DOI: $10.12731 / 2306-1561-2013-4-21$

\title{
MULTICRITERION TASK OF FORMING AN ENTERPRISE DEVELOPMENT STRATEGY
}

Moiseev A.N., Prikhodko M.V., Satyshev S.N., Hvoinsky L.A.

\section{Abstract}

In order to form an enterprise development strategy in the presence of multiple criteria derived from the expert survey is proposed to build a network intensive options that correspond to the plurality of Pareto. By building a network of intensive options, you can perform various tasks forming the control parameters with regard to the factors of cost and risk.

Keywords: strategy, multicriteriality, indexing, evaluation, aggregation, stressful option.

\section{УДК 681.3}

\section{МНОГОКРИТЕРИАЛЬНАЯ ЗАДАЧА ФОРМИРОВАНИЯ СТРАТЕГИИ РАЗВИТИЯ ПРЕДПРИЯТИЙ}

\section{Моисеев А.Н., Приходько М.В., Сатышев С.Н., Хвоинский Л.А.}

\section{Аннотация}

Для формирования стратегии развития предприятий при наличии множества критериев, полученных на основе экспертного опроса, предлагается построение сети напряженных вариантов, которые соответствуют множеству Парето. Построив сеть напряженных вариантов, можно решать различные задачи формирования параметров управления с учетом факторов стоимости и риска.

Ключевье слова: стратегия, многокритериальность, индексачия, оценки, агрегирование, напряженный вариант.

\section{Парето-оптимальные варианты}

Вариант $x$ является напряженным, если не существует другого варианта $y$, имеющего то же значение комплексной оценки, у которого оценки по всем критериям не выше, чем у варианта $x$. Так, вариант (рисунок 1) $x=(2,2,4)$, имеющий комплексную оценку $K=3$, не является напряженным, так как имеется вариант $y=$ $(2,2,3)$, имеющий такое же значение комплексной оценки и в то же время его оценки 
по критериям не превышают оценок варианта х. Для варианта $y=(2,2,3)$ таких вариантов не существует.

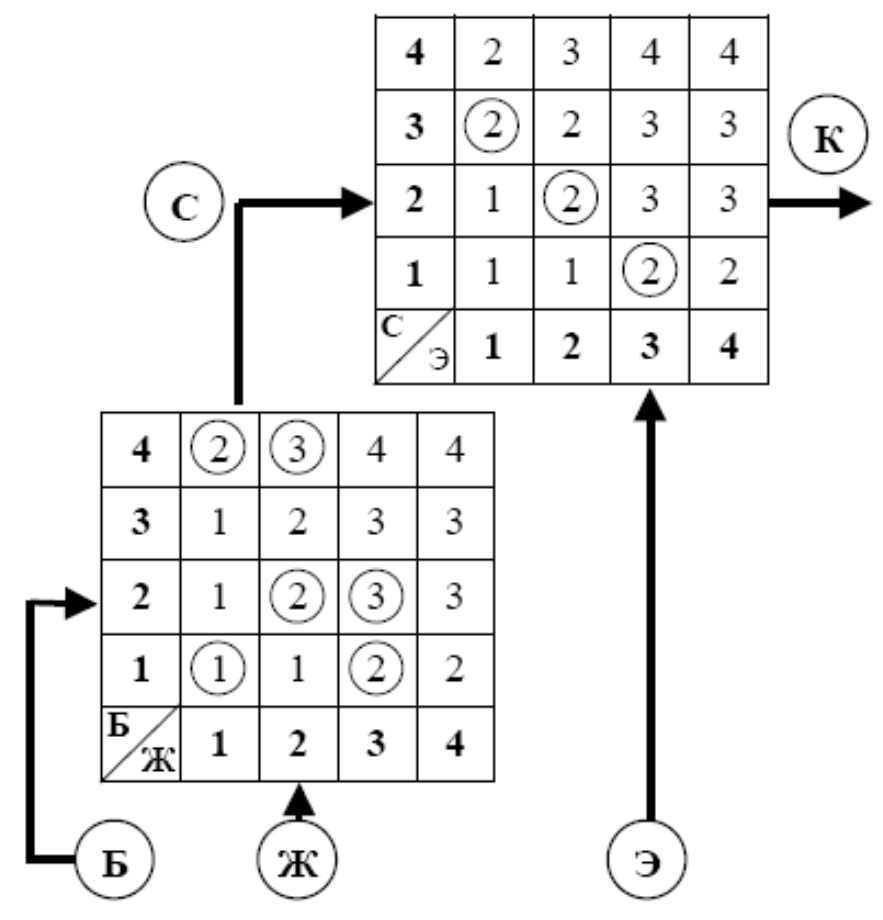

\section{Рисунок 1 - Схема формирования комплексной оценки}

Фактически, напряженные варианты это Парето-оптимальные варианты в пространстве критериев [1 - 11]. Таким образом, мы можем ограничиться рассмотрением только напряженных вариантов. Опишем алгоритм построения всех напряженных вариантов.

В его основе также лежит метод индексации вершин сети напряженных вариантов снизу вверх.

Первый шаг. Помечаем нижние вершины сети индексами sij.

Общий шаг. Вершины следующего (более высокого) уровня сети напряженных вариантов помечаются только после того, как помечены все смежные вершины нижележащего уровня. При этом, индекс вершины-квадрата (в таких вершинах записывается одно число - оценка соответствующего агрегированного критерия) равен минимальному из индексов смежных вершин-кружков нижележащего уровня, а индекс вершины-кружка (в кружке записаны два числа - это пара оценок критериев нижнего уровня, агрегирование которых дает соответствующую оценку критерия верхнего уровня) равен сумме индексов смежных вершин-квадратов нижележащего уровня.

Для получения какого-либо напряженного варианта поступаем следующим образом. Рассматриваем начальную вершину (вход) сети. Из нее исходят три дуги. Берем любую из них, например, дугу, ведущую в вершину (2; 2). Из вершины $(2 ; 2)$ исходят две дуги. Отмечаем обе эти дуги. Дуга, ведущая в вершину 2 по показателю «Э» указывает, что по этому показателю требуется достичь состояния 
«удовлетворительно». Дуга, ведущая в вершину 2 по показателю «С» указывает, что по этому показателю также требуется достичь состояния «удовлетворительно». Из трех вариантов достижения оценки 2 по показателю «С» выбираем любой (например, вариант $3 ; 1)$, что соответствует оценке «хорошо» по показателю «Ж» и оценке «плохо» по показателю «Б». Полученному напряженному варианту соответствует подграф сети, выделенный на рисунке 2 толстыми дугами. Он определяет напряженный вариант $(3 ; 1$; 2). Имея сеть напряженных вариантов, можно определить число напряженных вариантов, обеспечивающих получение требуемой оценки.

\section{Алгоритм индексации вершин}

Для этого предложен алгоритм индексации вершин сети:

Первый шаг. Помечаем конечные вершины сети индексами 1 (индексы указаны в верхней половине вершины).

Второй шаг. Двигаясь снизу вверх, последовательно помечаем все вершины. Индекс вершины-кружка на рисунке 2 равен произведению индексов смежных с ней двух вершин нижнего уровня. Индекс вершины-квадрата на рисунке 2 равен сумме индексов смежных с ней вершин нижнего уровня. Индекс начальной вершины-квадрата определяет число напряженных вариантов.

Обоснование алгоритма непосредственно следует из описанного способа определения индексов. Индексы вершин указаны на рисунке 2 в верхней части вершин $[6,7,11]$. Число напряженных вариантов равно шести.

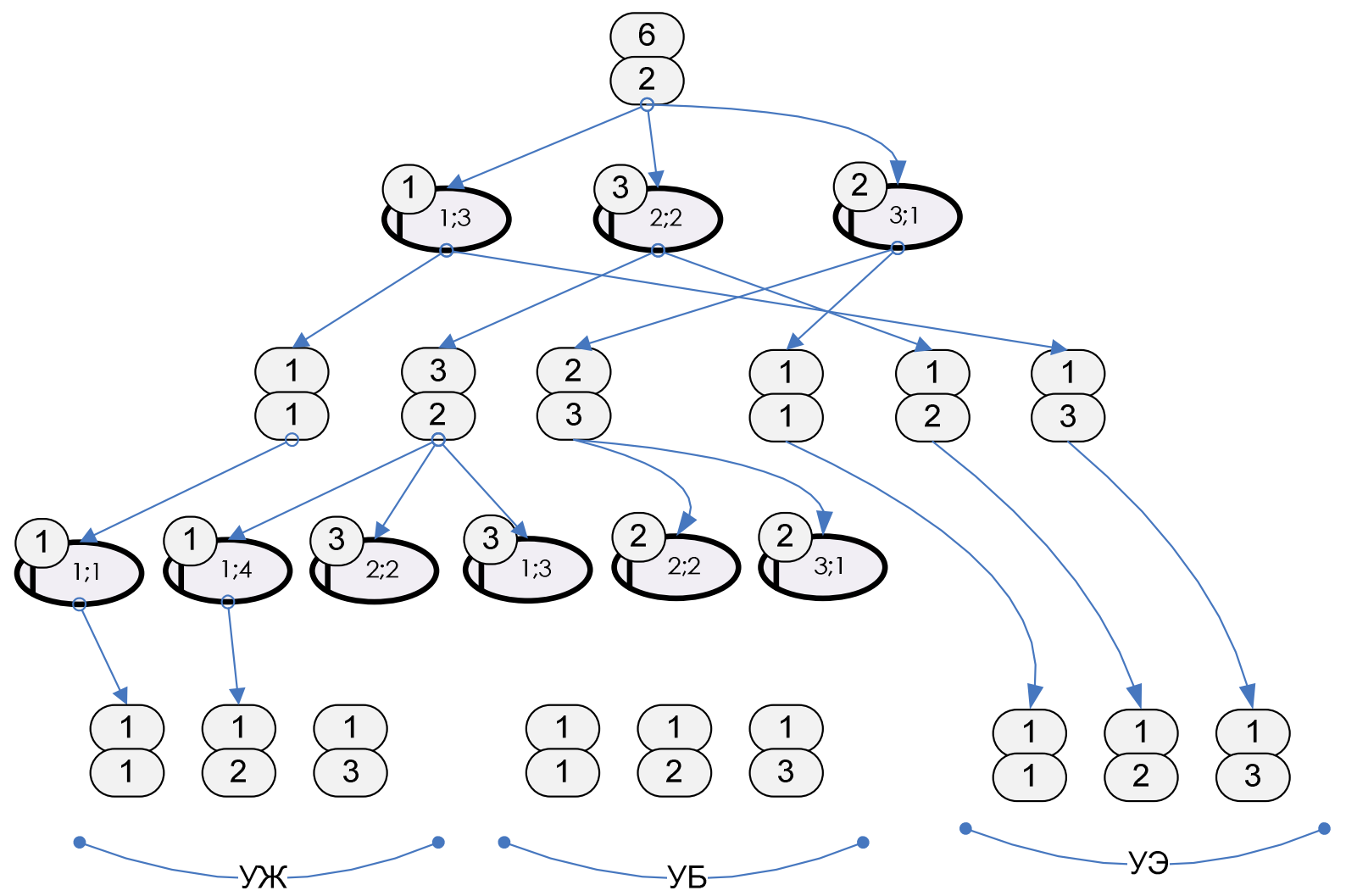

Рисунок 2 - Сеть напряженных вариантов 
Обобщением системы комплексного оценивания является система нечеткого комплексного оценивания, в которой оценки по каждому из критериев являются в общем случае нечеткими и агрегируются в соответствии с матрицами свертки. Нечетким оценкам могут соответствовать вектора степеней уверенности экспертов в достижении четких оценок. Получаемая в результате агрегирования оценка также является нечеткой и несет в себе больше информации.

Пусть $x_{1}$ - нечеткая оценка по первому критерию, задаваемая функцией принадлежности $\mu x_{1}(x l)$ на универсальном множестве, определяемом соответствующей шкалой, а $x_{2}$ - нечеткая оценка по второму критерию, задаваемая функцией принадлежности $\mu x_{2}\left(x_{2}\right)$.

$\mathrm{B}$ соответствии с принципом обобщения полученная в результате агрегирования по процедуре $\mathrm{f}(\bullet, \bullet)$, задаваемой матрицей свертки, нечеткая оценка $\mathrm{x}$ будет определяться функцией принадлежности:

$$
\mu_{x}(x)=\sup _{(x 1, x 2) / f(x 1, x 2)=x} \min \left[\mu_{x 1}\left(x_{1}\right), \mu_{x 2}\left(x_{2}\right)\right] .
$$

В предельном случае, то есть когда агрегируются четкие оценки, естественно, агрегированная оценка является четкой и совпадает с получающейся в результате использования четкой процедуры комплексного оценивания с логическими матрицами.

Пусть требуется оценить эффективность стратегии развития в условиях неопределенности (критерий $\mathrm{X}$, рисунок 3), который определяется уровнем экономического развития предприятия (критерий $\mathrm{X} 1$ ) и активностью производственной деятельности (критерий Х2). Уровень экономического развития в свою очередь определяется уровнем инвестиций (критерий X11) и средней заработной платой (критерий X12), а активность производственной деятельности - объемом заказов по 1-му направлению производственной деятельности (критерий X21), объемом заказов по 2-му направлению (критерий X22) и т.д. (без ограничения общности можно остановиться на двух направлениях производственной деятельности).

Пусть значения оценок по каждому критерию могут принимать конечное число значений (будем использовать четырехбальную шкалу: 1 - «плохо», 2 «удовлетворительно», 3 -«хорошо» и 4 - «отлично»).

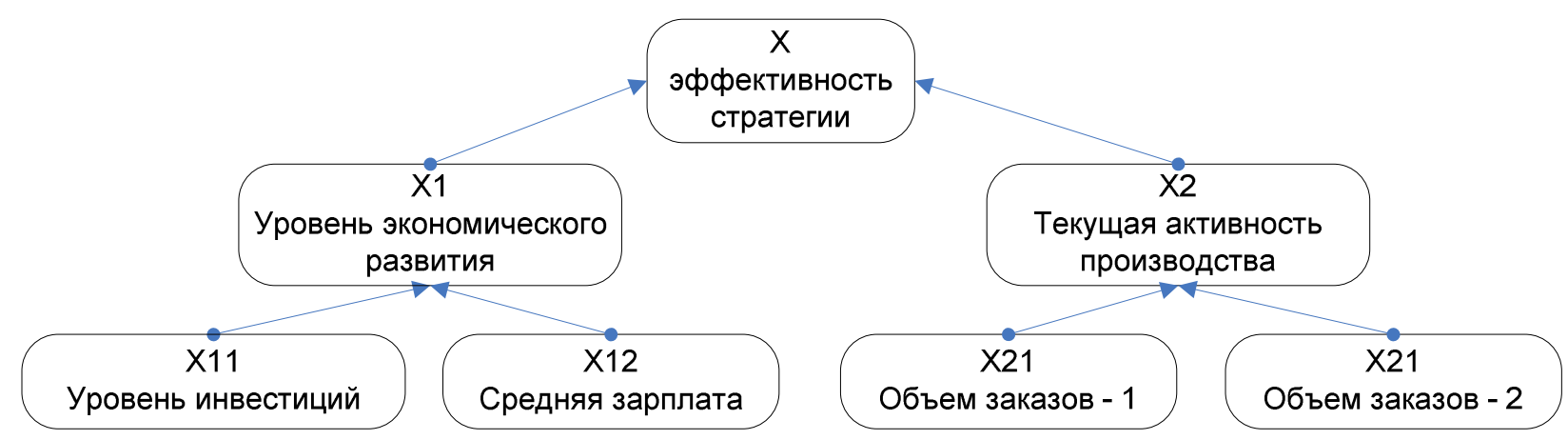

Рисунок 3 - Дерево критериев 
Требуется, имея оценки по критериям Х11, Х12, Х21, Х22 нижнего уровня, получить агрегированную оценку по критерию Х. В случае бинарного дерева для свертки критериев будем использовать матрицы свертки, значения элементов которых определяют агрегированную оценку при условии, что оценки по агрегируемым критериям являются номерами соответствующих строк и столбцов.

Пусть нечеткие оценки по критериям нижнего уровня принимают значения, приведенные в таблице 1. Используя матрицы свертки и выражение (1), получаем нечеткие оценки по агрегированным критериям.

Таблица 1 - Агрегирование нечетких оценок

\begin{tabular}{|l|l|l|l|l|}
\hline & \multicolumn{4}{|l|}{ Нечеткие значения } \\
\hline Критерии & 1 & 2 & 3 & 4 \\
\hline $\mathrm{X}$ & 0,00 & 0,20 & 0,70 & 0,30 \\
\hline $\mathrm{X} 1$ & 0,00 & 0,10 & 0,40 & 0,70 \\
\hline $\mathrm{X} 2$ & 0,20 & 0,90 & 0,30 & 0,10 \\
\hline $\mathrm{X} 11$ & 0,00 & 0,20 & 0,40 & 0,70 \\
\hline $\mathrm{X} 12$ & 0,00 & 0,10 & 1,00 & 0,40 \\
\hline $\mathrm{X} 21$ & 0,20 & 0,90 & 0,30 & 0,10 \\
\hline $\mathrm{X} 22$ & 0,00 & 0,30 & 0,95 & 0,40 \\
\hline
\end{tabular}

По аналогии с напряженными вариантами в системах четкого оценивания, можно рассматривать нечеткие напряженные варианты. Пусть задан нечеткий вектор оценок агрегированного критерия (в данном случае - это вектор $\mathrm{X}=(0 ; 0,2 ; 0,7 ; 0,3)$ ). Напряженными являются минимальные вектора агрегируемых оценок, приводящие к заданному нечеткому вектору агрегированных оценок. В данном случае - это вектора $\mathrm{X} 1=(0 ; 0 ; 0,2 ; 0,7)$ и $\mathrm{X} 2=(0,2 ; 0,7 ; 0,3 ; 0)$. Напряженному варианту будет соответствовать следующий набор значений оценок нижнего уровня: $\mathrm{X} 11=(0 ; 0 ; 0,2$; $0,7), \mathrm{X} 12=(0 ; 0 ; 0,7 ; 0), \mathrm{X} 21=(0,2 ; 0,7 ; 0,3 ; 0), \mathrm{X} 22=(0 ; 0 ; 0,7 ; 0)$.

Разности между приведенными в таблице 1 значениями оценок и напряженными можно считать резервами по соответствующим критериям, что позволяет ставить и решать задачи оптимизации резервов, затрат и риска. Таким образом, процедура комплексного оценивания является гибким и эффективным инструментом обработки информации, используемой при принятии управленческих решений.

\section{Список информационных источников}

[1] Солнцев А.А., Якунин П.С., Чичерин А.В., Кузнецов С.А. Моделирование транспортной системы на основе гибридного автомата. / Методы управления потоками в транспортных системах: сб. науч. тр. МАДИ. Ротапринт МАДИ. - М., 2009 . - C. $41-45$

[2] Катырин С.Н., Солнцев А.А., Тимофеев П.А., Якунин П.С. Агрегированные критерии эффективности бизнес-процессов управления производственным циклом промышленных предприятий. // Вестник МАДИ. - М.: МАДИ, 2011. Вып. 4 (27). - C. 53-59. 
[3] Николаев А.Б., Брыль В.Н., Кузнецов С.А. Теоретические основы решения информационно-сложных задач. //Вестник Московского автомобильно-дорожного государственного технического университета (МАДИ). - 2010. - №4. - С74-78.

[4] Николаев А.Б., Юрчик П.Ф., Голубкова В.Б., Берко Н.А. Двухуровневая иерархическая система координации технологического процесса строительства нефтепроводов и объектов газотранспортного комплекса. // Вестник Московского автомобильно-дорожного государственного технического университета (МАДИ). - 2011. - №2. - С54-57.

[5] Николаев А.Б., Брыль В.Н. Проблемы решения информационно-сложных задач. //Вестник Московского автомобильно-дорожного государственного технического университета (МАДИ). - 2008. - №3. - С78-82.

[6] Солнцев А.А. Модели нечеткого ситуационного анализа при описании вложенных процессов многоцелевой деятельности дилерской сети // Автоматизация и управление в технических системах. - 2013. - № 1(3); URL: auts.esrae.ru/3-71 (дата обращения: 19.11.2013).

[7] Остроух А.В. Информационные технологии в научной и производственной деятельности / [ред. А.В. Остроух] - М: ООО "Техполиграфцентр", 2011. - 240 с. ISBN 978-5-94385-056-1.

[8] Куфтинова Н.Г. Процессно-ориентированный подход к автоматизации планирования и управления транспортировкой продукции предприятий промышленности / А.В. Остроух, Н.Г. Куфтинова // Вестник МАДИ - 2010. - Вып. 4(23). - C. 62-66.

[9] Остроух А.В. Исследование начального периода моделирования на точность среднеинтегральной оценки имитационных моделей / А.В. Остроух, А.А. Солнцев, Н.В. Солдатов, К.А. Новицкий, П.С. Якунин // Вестник МАДИ - 2010. Вып. 2(21). - С. 61-65.

[10] Остроух А.В. Математическая модель связей в системе диагностики электрооборудования автомобилей / А.В. Остроух, А.А. Солнцев, О.Ф. Калухов, Г.Г. Ягудаев // Вестник МАДИ - 2010. - Вып. 2(21). - С. 66-70.

[11] Приходько В.М., Солнцев А.А., Саная А.Г. Сетевая теоретико-игровая модель рациональных закупок в задаче формирования адаптивного механизма согласованных цен в схеме снабжения дилерской сети // Автоматизация и управление в технических системах. - 2013. - № 1(3); URL: auts.esrae.ru/3-70 (дата обращения: 05.09.2013). 